\title{
Scanning thermal microscopy on samples of varying effective thermal conductivities and identical flat surfaces
}

\author{
E. Guen ${ }^{1}$, P-O. Chapuis ${ }^{1}$, R. Rajkumar ${ }^{3}$, P. S. Dobson ${ }^{2}$, G. Mills ${ }^{3}$, J. M. R. Weaver ${ }^{2}$ and S. Gomés ${ }^{1}$ a) \\ ${ }^{1}$ CETHIL UMR5008, Univ Lyon, CNRS, INSA-Lyon, Université Claude Bernard Lyon 1, F-69621, Villeurbanne, France \\ ${ }^{2}$ Univ Glasgow, School of Engineering, Glasgow, Scotland, G12 8QQ, United Kingdom \\ ${ }^{3}$ Kelvin Nanotechnology, Glasgow, Scotland, G12 8LS, United Kingdom \\ a)Author to whom correspondence should be addressed: severine.gomes@insa-lyon.fr
}

\section{ABSTRACT}

We propose an approach for the characterization of scanning thermal microscopy ( $\mathrm{SThM}$ ) probe response using a sample with silicon dioxide steps. The chessboard-like sample provides a series of nine surfaces made of the same material, with identical roughness, but consisting of different thicknesses of silica layers standing on a single silicon wafer. The nine regions have different effective thermal conductivities, allowing calibration of SThM probes within a given set of surface conditions. A key benefit is the possibility of comparing the spatial resolution and the sensitivity to vertical inhomogeneities of the sample for different probes. A model is provided to determine the thermal contact area and contact thermal resistance from the experimental data. The results underline that ballistic heat conduction can be significant in crystalline substrates below the top thin films, especially for film thicknesses lower than $200 \mathrm{~nm}$ and effective thermal contact radius lower than $200 \mathrm{~nm}$. They also highlight the sensitivity of SThM to ultrathin films, as well as the substrate below micrometric films under in-air conditions but not when in vacuum. This work advances quantitative nanometer-scale thermal metrology, where usual photothermal methods are more difficult to implement.

\section{INTRODUCTION}

Thermal effects at the micro and nanoscale are very different from those at the macroscopic scale, and are of profound fundamental and practical importance for nanoscience and nanotechnology. The measurement of thermal effects at small scales has driven the development of new instrumentation and experimental methods since early steps taken 35 years ago. Such instrumentation is needed both to elucidate the mechanisms of heat transport and as prototypes of utilitarian measurement systems. Many techniques have been developed for exploring the thermal properties of materials on a small scale, including electrical-based ones such as the $3 \omega$ method [1] and bridge-based ones involving self-heating of the nanostructures [2]. Optical methods such as thermo-reflectance [3] and Raman spectroscopy [4], have limited lateral spatial resolution because of the diffraction of light. To overcome this spatial resolution issue, three options are possible. The first one is to deposit a thin film of nanometric lateral extent at the surface of the sample and illuminate it at a wavelength where it selectively absorbs, therefore heats, while the sample itself does not absorb [5]. The analysis of the temperature of the deposited element can then provide information on the sample thermal properties. However, this process is rather complex, limited to certain classes of samples, and alters the sample. The second option is to use scanning probe microscopy (SPM)-based techniques [6-8]. Mainly based on atomic force microscopy (AFM), modern scanning thermal microscopy (SThM) can reach lateral resolutions of a few tens of nanometers when employing nanoprobes operated under vacuum [9]. The method has already made possible the thermal investigation of bulk and thick films (thickness larger than $20 \mu \mathrm{m}$ ) [10-12], thin films and 2D materials [10,12-13], suspended membranes [14] and 1D nanostructures such as nanowires [15].

The principle of thermophysical property measurement by SThM is based on the control of the thermal interaction between a small self-heated probe located close to or in contact with the surface of the sample to be studied. The most popular thermal probes are equipped with a resistive element at the tip apex, which in the socalled 'active mode' simultaneously acts as a sensor and a heat source for the sample in order to determine its local thermal properties. Sufficient dc or ac current is passed through the probe to produce significant self-heating by the Joule effect. When the probe is in contact with a specimen, the heat flow passing from the tip into the sample is affected by the local thermal conductance of the sample, leading to a modification of the probe temperature and consequently of its electrical resistance. On mechanically scanning the probe over the sample the local variations of sample thermal conductance are used to obtain the contrast in the thermal image and to study heat transport within the sample.

Using this approach, determination of the thermal properties of a sample is not direct, rather a "direct measurand" (i.e. a quantity [16] which is related to the heat transfer between probe and sample) must first be obtained. Therefore, the SThM technique, when applied to thermal property determination, requires inverse methods. The whole procedure for thermal conductivity measurement using SThM includes a number of steps. The first is the definition of a relevant direct measurand. The second one is to find reference samples whose thermal properties are well-known. The direct measurand is obtained on these samples and then plotted against the sample thermal conductivity. The third step is the development of a modelling 
method to fit the experimental data and provide a calibration curve. To determine the thermal conductivity of an unknown sample, its direct measurand must be determined and compared with the calibration curve. This employs inversion of the model to retrieve the corresponding value of thermal conductivity.

Based on this scheme, different strategies have been proposed. The direct measurand is either the change in probe electrical resistance (for resistive SThM probes $[14,17]$ ) or the variation of derived parameters such as the probe voltage [18] or the change in electrical power dissipated in the probe [19] while the probe is first held out of contact and then in contact with the sample. Samples used for probe calibration are bulk samples having well-known and different values of thermal conductivity $[12,19]$. An issue is that the thermal contact and subsequently its thermal resistance strongly depend on parameters of the sample surface such as roughness [20], presence of a native oxide [6] and on the thermal resistance of the tip-sample contact [6], all of which change for each reference sample. Consequently, it would be better to use a different approach for determining a calibration curve for thermal conductivity measurements performed in air, even in this "simple" case of bulk materials. While the previous approach may be sufficient for characterizing bulk samples of similar surface states or thermalconductivity ranges less sensitive to the surface states, it is clearly insufficient for characterizing more complex samples. This is especially true for nanomaterials, where a certain degree of modelling is required due to the absence of sample with the exact same architecture for calibration. Analytical modeling [19-22], used for fitting experimental data relative to these samples, is based on the analysis of the thermal balance of the "probe-sample-environment" system while the probe is either out of contact or in contact with the sample. The heat flux transferred from the heated probe to the sample is then described using a thermal resistance network accounting for the thermal contact and the heat spreading within the sample. Such approaches are often based on the assumptions that the thermal contact radius and the contact thermal resistance are invariant with the sample thermal conductivity and no ballistic thermal transport effects operate within the samples [6]. However, it has been shown that the thermal contact radius and the contact thermal resistance vary as a function of the sample thermal conductivity when measurements in and out of contact with the sample are performed in air [17, 19, 23-26]. Moreover ballistic effects are expected to operate, specifically for high thermal conductivity crystalline samples, when the thermal contact radius reaches values of the same magnitude or lower than the averaged mean free path of energy carriers in the sample material [6]. A conclusion of all these different points is that an improved, reliable calibration technique is required.

The method described here provides the measurand as a function of an effective thermal conductivity, with reference bulk samples of known thermal conductivity and similar roughness [6] setting limits on the thermal conductivity to be inferred from the calibration. This method substantially removes the dependence of the estimated value of thermal conductivity on errors that exist in estimates of the thermal contact radius and resistance. It consists first of assessing a sample designed specifically for the calibration of SThM probes. Based on steps of silicon dioxide on a silicon substrate, the sample presents areas with different effective thermal conductivity on its surface. The surface material is the same everywhere, with a measured sample roughness lower than $0.8 \mathrm{~nm}$, ensuring invariance of the mechanical contact between the probe and the sample. For sample analysis a model describing heat spreading from a localized heat source at the sample surface in the $\{$ thin film+substrate $\}$ system is proposed, which can also be useful for use with optical methods involving heated elements of small lateral extension. Here, both the sample and model are used to calibrate two types of SThM probes: a "Wollaston" probe [27] and a "Pd" probe [28]. Section 2 of this paper describes the sample and the specifications, and introduces the model, describing heat conduction within the sample, generalizing previous SThM studies of thin films [29]. Section 3 summarizes the setup and measurement methodology used. In Section 4 the results obtained are analyzed for the two types of probe. A summary of the main findings and a discussion regarding the application of the proposed sample for probe calibration in SThM precede the conclusion of the paper.

\section{SAMPLE}

\section{A. Sample description and characteristics}

The fabricated sample consists of steps of wet thermally grown silicon dioxide $\left(\mathrm{SiO}_{2}\right)$ with different thicknesses as defined by successive steps of photolithography and wet etching using buffered HF. The substrate is a $380 \mu \mathrm{m}$ thick (100) oriented silicon wafer ndoped with phosphorus having a nominal electrical resistivity of 0.1 $10 \Omega . c m$. The sample has nine steps of different $\mathrm{SiO}_{2}$ thickness ranging from a few nanometres to $1000 \mathrm{~nm}$ (Table I). As shown in Fig. 1 patterns are squares of $30 \mu \mathrm{m}$ of width forming a mosaic of $(90 \mu \mathrm{m} \times 90 \mu \mathrm{m})$ which can be completely analyzed from a single SThM image. The width of squares were chosen so that each is larger than the largest thermal contact radius obtained for the Wollaston probe, the largest SThM probe used in this work. Specifically, this largest thermal contact radius was found to be about $10 \mu \mathrm{m}$ for a polymeric sample in the frame of measurements performed under ambient air conditions.

Thermo-reflectance microscopy was used elsewhere [30] to measure the thermal conductivity $k_{\mathrm{SiO} 2}$ of the sample, providing an intrinsic conductivity value for the $\mathrm{SiO}_{2}$ as well as the thermal resistance at the boundary $r_{t b r}$ between the $\mathrm{SiO}_{2}$ film and the $\mathrm{Si}$ substrate. Values determined are $k_{\mathrm{SiO} 2}=1.1 \mathrm{~W} \cdot \mathrm{m}^{-1} \cdot \mathrm{K}^{-1}$ and $r_{t b r}=4.4$ $10^{-8} \mathrm{~m}^{2} . \mathrm{K} \cdot \mathrm{W}^{-1}$.

\section{B. Sample modelling}

In all experiments, a temperature profile is imposed on the top surface. It is common practice to replace the profile by an average temperature in order to simplify the reasoning.

(i) Diffusive regime. For an isothermal discoidal heating source on a thin layer with perfect contact to a thick (assumed semi-infinite) underlying substrate $\left(r_{t b r}=0\right)$ (Fig.2), the thermal resistance can be described using the model of Yovanovich et. al. [31] based on Dryden's work. The in-plane temperature $\theta(r, z)$ relative to the ambient temperature is given by: 


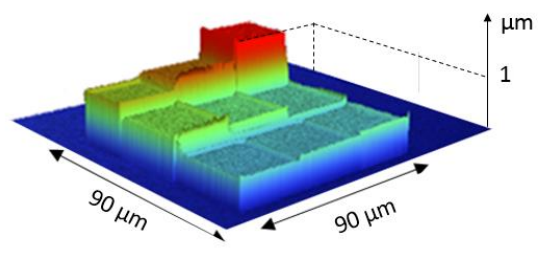

(a)



(b)

FIG. 1. (a) White light interferometry topography 3D-image and (b) atomic force microscopy topography 2D-image of the $\mathrm{SiO}_{2}$ pattern designed for SThM measurements.

Table I. Thickness and roughness of each of the steps as measured by AFM.

\begin{tabular}{llllllllll}
\hline $\begin{array}{l}\text { Step } \\
\text { number }\end{array}$ & 1 & 2 & 3 & 4 & 5 & 6 & 7 & 8 & 9 \\
\hline $\begin{array}{l}\text { Thickness } \\
(\mathrm{nm})\end{array}$ & 7 & 12 & 30 & 65 & 145 & 237 & 330 & 530 & 950 \\
$\begin{array}{l}\text { Rms } \\
\text { roughness } \\
(\mathrm{nm})\end{array}$ & 0.12 & 0.65 & 0.54 & 0.65 & 0.64 & 0.75 & 0.39 & 0.18 & 0.13 \\
\hline \hline
\end{tabular}



FIG. 2. Representation of the layer with thermal conductivity $k_{\text {film }}$ and thickness $t$ on a thick substrate with thermal conductivity $k_{\text {sub. }}$

$$
\theta(r, z)=\frac{Q}{2 \pi k_{f i l m} b} \int_{0}^{\infty}\left[\frac{\exp \left(-\frac{\zeta z}{b}\right)+K \exp \left(\frac{\zeta}{b}(z-2 t)\right)}{1-K \exp \left(-\frac{2 \zeta t}{b}\right)}\right] J_{0}\left(\zeta \frac{r}{b}\right) \frac{\sin (\zeta)}{\zeta} d \zeta(1)
$$

where $r$ is the distance from the source centered on $r=0$ in the source plane, $z$ is the distance from the source located in $z=0$ in the cross- plane direction, $Q$ is the thermal flow, $J_{0}$ is a Bessel's function of order 0 and $K$ is defined as

$$
K=\frac{1-\frac{k_{\text {sub }}}{k_{\text {film }}}}{1+\frac{k_{\text {sub }}}{k_{\text {film }}}}
$$

The thermal resistance across the film and substrate is defined by Dryden [32]:

$$
R_{f i l m+s u b}=\frac{\theta_{\max }}{Q},
$$

where $\theta_{\max }=\theta(r, 0), 0<r<b$. It is given by:

$$
R_{\text {film }+ \text { sub }}=\frac{1}{\pi k_{\text {film }} b} \int_{0}^{\infty}\left[\frac{1+K \exp \left(-\frac{2 \zeta t}{b}\right)}{1-K \exp \left(-\frac{2 \zeta t}{b}\right)}\right] J_{1}(\zeta) \frac{\sin (\zeta)}{\zeta^{2}} d \zeta
$$

where $J_{l}$ is a Bessel's function of the first kind. Accounting for the thermal boundary resistance $r_{t b r}$ at $z=t$ can be achieved using an effective thickness $t_{\text {eff }}$ as described by Menges et al. and Muzychka et al. [33, 34]:

$$
t_{\text {eff }}=t+k_{f i l m} \cdot r_{t b r}
$$

The rationale behind this expression is that the boundary resistance modifies the flux lines in the film.

Let us now consider a few asymptotic results. For the bulk case (homogeneous isotropic material with a thermal conductivity $k=$ $\left.k_{f i l m}=k_{s u b}\right)$ with an isothermal heat source on the surface, Eq. (4) reduces to

$$
R_{S-b u l k}=\frac{1}{4 . k \cdot b} .
$$

Note that the result for the isothermal hypothesis deviates by about $8 \%$ from the result for the iso-flux source hypothesis, for which the thermal resistance in the sample is $R_{s-b u l k}=\frac{8}{3 . \pi^{2}} \frac{1}{k \cdot b}$. As discussed at the beginning of the section, this can lead to some error in the model. However, if the experimental temperature profile on the top surface is known, the error is cancelled by averaging the temperature over the source. The exact bulk thermal resistance lies between these two values. For a film thickness smaller than the size of the heated disk, such that $t / b \rightarrow 0$, Eq. (1) leads to

$$
R_{f i l m+s u b} \rightarrow \frac{1}{4 \cdot k_{s u b} \cdot b}
$$

which indicates that the film thermal resistance becomes negligible in comparison to that of the substrate.

(ii) Heat conduction involving ballistic dissipation. The previous modeling is commonly used in SThM for the analysis of thin layers on a substrate whatever the materials involved. However, it applies only to purely diffusive thermal transport and does not account for size effects related to the sub-micrometric/nanometric heat sources relevant to SThM vacuum experiments. In our sample, heat transport in $\mathrm{SiO}_{2}$ is diffusive. However, the $\mathrm{Si}$ substrate is crystalline, with an inelastic mean free path for acoustic phonons on 
the order of $\Lambda_{\text {sub }}=170 \mathrm{~nm}$ at ambient temperature $T_{a}$ [35]. Ballistic heat conduction can therefore occur in the substrate, at least partly, if the heated region at the oxide-substrate boundary is of same magnitude as $\Lambda_{s u b}$ or lower than this value. An expression generalizing Eq. (1) to heat dissipation without assumption on the thermal transport regime would be helpful. To do so, it is key to analyze the heated region at the oxide-substrate boundary. Fig. 3 reports temperature profiles calculated by Eq. (1) at the sample surface and at the thin film/substrate boundary $(z=t)$ for two heat source radii $b(100$ and $300 \mathrm{~nm})$ and two film thicknesses $t$ (237 and $65 \mathrm{~nm})$. Thermoreflectance data was used for the the silicon dioxide film thermal conductivity and the thermal resistance at the boundary with silicon [30]. Fig. 3(a) shows a case where $b(100 \mathrm{~nm})$ is smaller than the thickness of the film $t(237 \mathrm{~nm})$. Conversely, Fig. 3(b) shows a case in which $b(300 \mathrm{~nm})$ is greater than $t(65 \mathrm{~nm})$. The temperature profile at the film/substrate boundary (full blue line) is steeper for the lowest thickness, indicating that heat does not spread easily in this thin film. In both cases, the heated disc at the oxide-substrate boundary is of the order of the phonon mean free path.

In order to account analytically for the partly-ballistic dissipation in the substrate, we rely on Wexler's approach [36], where thermal resistance is the sum of the diffusive (Fourier, Eq. (6)) and ballistic (Sharvin [37]) resistances. It was shown numerically that the maximal error induced by this approach is $11 \%$ [38]. The ballistic thermal resistance, in the case of the averaged mean free path, is given by [36]:

$$
R_{\text {bal }}=\frac{4}{3 \cdot \pi \cdot b^{2}} \frac{\Lambda_{\text {sub }}}{k_{\text {sub }}}
$$

However, the Wexler interpolation summing Eq. (6) and $R_{b a l}$ was applied for semi-infinite media with isothermal discoidal sources of radius $b$ ', which is not the case for the oxide-substrate boundary as shown by Fig. 3. An effective radius $b^{\prime}$ can be obtained as follows. First, the thermal resistance in the substrate is determined by dividing the flux by the average temperature at the oxide-substrate boundary:

$$
R_{\text {sub }}=\frac{1}{\pi k_{\text {film }} b^{\prime}} \int_{0}^{\infty} \frac{(K+1) \exp \left(-\frac{\zeta t}{b}\right)}{1-K \exp \left(-\frac{2 \zeta t}{b}\right)} J_{1}\left(\zeta \frac{b^{\prime}}{b}\right) \frac{\sin (\zeta)}{\zeta^{2}} d \zeta
$$

Then, this expression is equated with $\frac{1}{4 \cdot k_{s u b} . b}$, which describes heat spreading in the substrate by means of diffusion. The effective isothermal radius $b$ ' can be plotted by keeping the same average temperature in the plane (shown in Fig. 3 using the blue dotted line termed "simplified"). The result of the numerical determination of $b^{\prime}$ ' is shown in Fig. 4(a). For the lowest values of both $t$ and $b, b^{\prime}$ may be of the order or less than the averaged phonon mean free path in the substrate $\Lambda_{s u b}$. The maximum relative difference between the "real" temperature and the idealized isothermal profile, observed in Fig. 3(a,b) for two special cases, is studied as a function of the $b / t$ ratio in Fig. 4(b). It appears that for large thicknesses (vs $b$ ), the maximum relative difference can be as high as $40 \%$. It takes a $b / t$
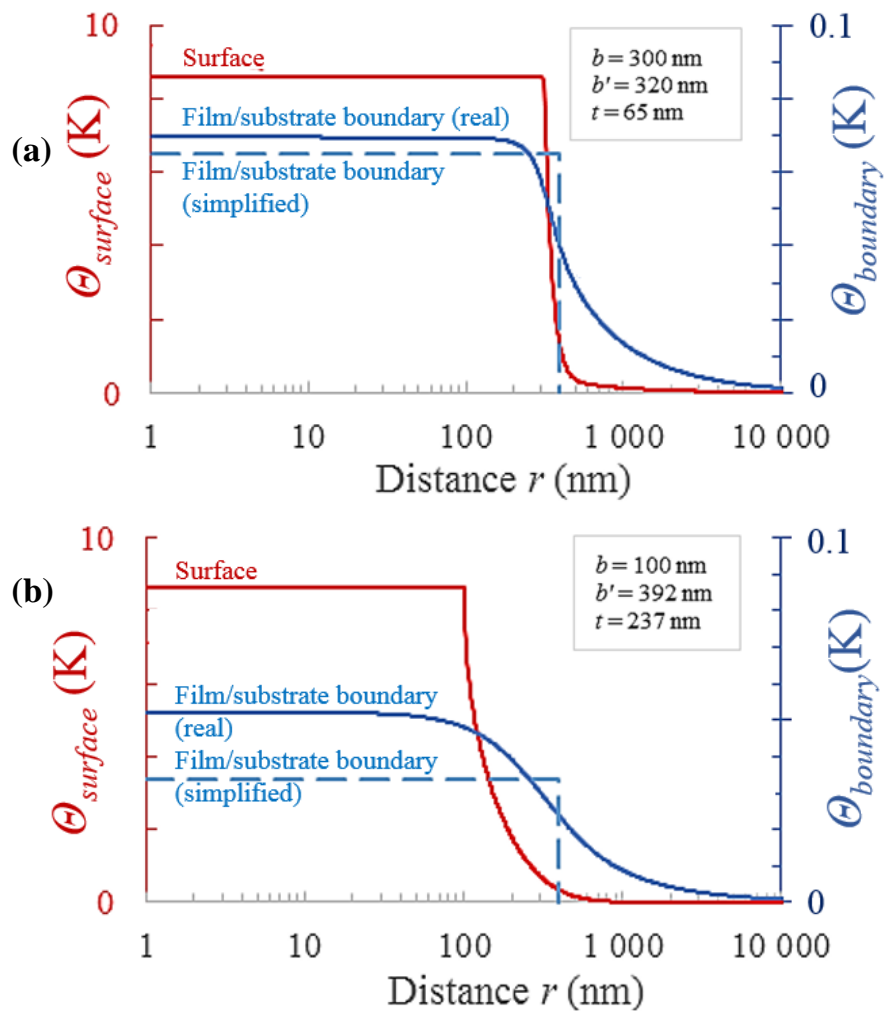

FIG. 3. Temperature profile at sample surface and at film/substrate interface for two $b$ thermal radius configurations compared with the film thickness $t$. (a) $b>t$, with $b=100 \mathrm{~nm}, t=237 \mathrm{~nm}$, (b) $b<t$, with $b=300 \mathrm{~nm}, t=65 \mathrm{~nm}$.

ratio greater than 3 to achieve a maximum relative deviation of less than $10 \%$.

The impact of ballistic transport in the substrate on the flux lines in the film is difficult to assess. There are two options: (a) one can treat ballistic resistance to the flow as an additional boundary resistance at the oxide-substrate boundary or (b) one can add the resistance to the volume resistances. (a) assumes a strong impact on the shape of the flux lines, expanding them and therefore modifying the size of $b^{\prime}$, while (b) is based on the determination of $b^{\prime}$ in the diffusive regime, as explained earlier. In the following, we will deal with assumption (b). The thermal resistance in the thin film $\left(R_{f i l m}\right)$ can be calculated from the subtraction of Eq. (9) from Eq. (4):

$$
R_{\text {film }}=R_{\text {film }+ \text { sub }}-R_{\text {sub }} \text {. }
$$

The thermal resistance at the boundary, which depends on the sample fabrication process and contacting materials, is simply obtained by integrating over the area of the spot: $R_{t b r}=\frac{r_{t b r}}{\pi \cdot b^{\prime 2}}$. Finally, the thermal resistance of the sample can be written as follows: 


$$
R_{s}=R_{\text {film }}+R_{\text {tbr }}+R_{\text {sub-ballistic }}+R_{\text {sub-diffusive }},
$$

where $R_{\text {sub-diffusive }}=\frac{1}{4 . k_{\text {sub }} \cdot b^{\prime}}$ and $R_{\text {sub-ballistic }}$ is given by Eq. (8). Fig. 5 represents the thermal resistances $R_{\text {film }}, R_{t b r}, R_{\text {sub-ballistic }}$ and

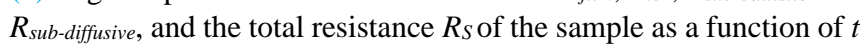
for a heat source radius on the surface $b=100 \mathrm{~nm}$. It is observed that for the lowest thicknesses, i.e. $t<b$ with $b=100 \mathrm{~nm}$, the thermal resistances $R_{\text {film }}, R_{t b r}$ and $R_{\text {sub-ballistic }}$ are very close and all contribute to the total resistance. In this regime, $R_{\text {sub-diffusive }}$ may be neglected at the first order. Then, for $t \geq 100 \mathrm{~nm}$, as the thickness of the layer increases, the decrease of $R_{\text {sub-diffusive, }} R_{t b r}$ and $R_{\text {sub-ballistic }}$ is significant. The thermal resistance of the sample then becomes dominated by that of the thin layer of low thermal conductivity. It is key to note therefore that the diffusive contribution of the substrate does not contribute significantly over the whole range of thicknesses. It is only if the heat source is larger that its contribution becomes more important.


FIG. 4. (a) Thermal radius at the film/substrate boundary $b^{\prime}$ as a function of the film thickness, for various heat source radii $b$. The zone where significant ballistic effect is expected is highlighted. (b) Maximum relative difference between the "real" temperature and the temperature calculated by considering a fixed thermal radius at the film/substrate interface as a function of the $b / t$ ratio.

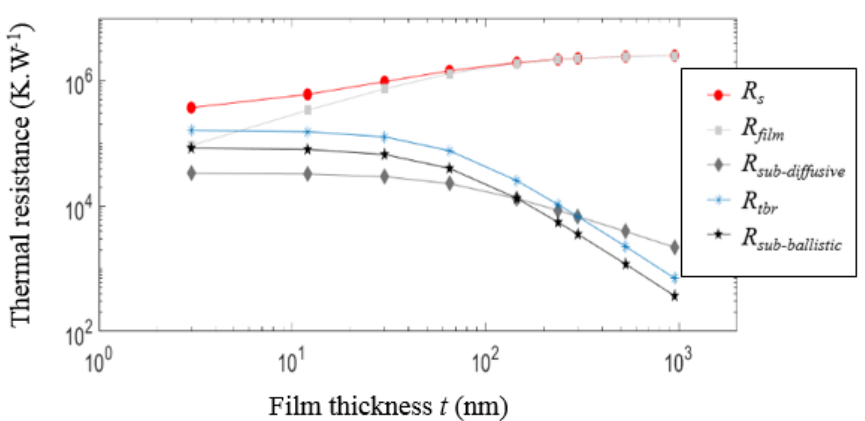

FIG. 5. Calculated thermal resistance of the structured sample $R_{s}$ for a discoidal heating source of radius $b=100 \mathrm{~nm}$ on the surface of the sample and consisting of the sum of four thermal resistances, that of thin film $\left(R_{f}\right)$, that due to the film/substrate boundary $\left(R_{t b r}\right)$ and the contributions of the substrate in the diffusive ( $R_{\text {sub-difusive }}$ ) and ballistic ( $R_{\text {sub-ballistic }}$ ) regimes.

\section{SThM MEASUREMENTS}

\section{A. Set-up, SThM probes and their calibration}

SThM measurements were made using an Atomic Force Microscopy based technique in a commercial instrument (NTEGRA-Aura AFM from NT-MDT). Two commercially available resistive SThM probes were used: a Wollaston wire probe and a Pd probe from Kelvin NanoTechnology (KNT). Fig. 6 shows scanning electron microscopy (SEM) images of both probes. The sensitive part of the Wollaston probe (Fig. 6(a)) comprises a Vshaped $\mathrm{Pt}_{90} / \mathrm{Rh}_{10}$ wire probe of $5 \mu \mathrm{m}$ in diameter and $200 \mu \mathrm{m}$ in length [27].

The $\mathrm{Pt}_{90} / \mathrm{Rh}_{10}$ wire probe is obtained by removing the silver shell from a Wollaston wire of $75 \mu \mathrm{m}$ cladding diameter. The cantilever is made of non-etched Wollaston wire. This fabrication process reveals many grooves on the $\mathrm{Pt}{ }_{90} / \mathrm{Rh}_{10}$ surface $[23,39]$. Consequently, when the probe apex contacts the sample, mechanical contact is established only between one of these grooves and the sample surface. The equivalent curvature radius of an individual groove is found to be several hundred nanometers [3]. The spring constant of the probe used was calculated to be $k_{r}=5 \pm 1 \mathrm{~N} \cdot \mathrm{m}^{-1}$ from the geometrical and physical parameters of the probe [39]. The temperature coefficient of electrical resistance $\alpha=\frac{1}{T_{p}} \frac{d R}{d T}$ of the overall probe was measured to be approximately $1.4 \times 10^{-3} \cdot \mathrm{K}^{-1}$. This was obtained from measurements of the electrical resistance of the probe, $R$, in an oven at different temperatures. Knowing $\alpha_{p}=1.66 \times 10^{-3} \mathrm{~K}^{-1}$ for the $\mathrm{Pt} 90 / \mathrm{Rh} 10$ wire part of the probe [39], the variation of electrical resistance $R$, which is due to that of the thermal sensor at the apex, can be related to the mean sensor temperature $\overline{T_{p}}$ :

$$
\Delta R=R-R_{0}=R_{p 0} \alpha_{p}\left(\overline{T_{p}}-T_{a}\right)=R_{p 0} \alpha_{p} \bar{\theta}_{p}
$$

where $R_{0}=3.00 \Omega$ is the total electrical resistance of the probe at room temperature $T_{a}, R_{p 0}=2.51 \pm 0.40 \Omega$ is the electrical resistance of the sensitive part of the probe at $T_{a}$ obtained from $3 \omega$ method application [40, 41] and $T_{a}=30^{\circ} \mathrm{C}$. 

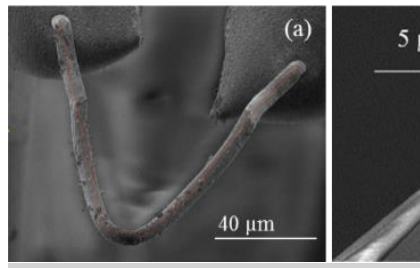

$5 \mu \mathrm{m}$



.

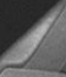
.

(b)
FIG. 6. SEM images of (a) the Wollaston probe and (b) the Pd probe.
The Pd probe comprises a thin resistive Pd film and pads of gold deposited on a silicon nitride $\left(\mathrm{Si}_{3} \mathrm{~N}_{4}\right)$ cantilever (Fig. 6(b)). Due to the shape and configuration of the probe apex, the contact with the sample is established through the $\mathrm{Si}_{3} \mathrm{~N}_{4}$ part of the probe only. The apex radius of curvature is smaller than $100 \mathrm{~nm}[28,42]$ and was estimated to be $50 \mathrm{~nm}$ using a methodology proposed in references $[43,44]$. The spring constant of the probe was measured to be $0.09 \pm 0.02 \mathrm{~N} \cdot \mathrm{m}^{-1}$ using the reference lever technique [45]. The overall temperature coefficient $\alpha$ of the Pd probe was determined to be $\alpha=7.8 \pm 0.1 \times 10^{-4} \mathrm{~K}^{-1}$ using the same method as previously specified for the Wollaston probe. Knowing $\alpha_{p}=1.2 \times 10^{-3} \mathrm{~K}^{-1}$ for the $\mathrm{Pd}$ wire and those of the other metallic components of the probe, the variation of the electrical resistance of the Pd film, which is the thermal sensor, can be estimated as a function of the film mean temperature. We find $R_{p 0}=63.95 \pm 12.79 \Omega$ at $T_{a}$ for the Pd probe used while $R_{0}=313,89 \Omega$.

Experimentally a home-made thermal control unit based on a balanced Wheatstone bridge was used to monitor the probe mean temperature $\overline{T_{p}}$ and the electrical power $P_{e l}$ dissipated in the probe.

\section{B. Measurement methodology and modeling}

For every sample studied the probe was moved from a position out of contact with the sample (probe-sample distance of $2 \mathrm{~mm}$ ) to position in contact, varying the mean probe temperature relative to the ambient temperature $\theta$. Knowing the power input $P_{e l}=R_{p} I^{2}(I$ is the current), the change in mean probe temperature was used to determine the variation of thermal conductance $\Delta G_{p-s}$ of the probesample system (see Fig. 7). Note that it was verified that this is similar to keeping temperature constant while monitoring $\Delta P_{e l}$.

From this measurement $\Delta G_{p-s}$ is associated with the global probe-sample heat transfer and is given by:

$$
\Delta G_{p-s}=G_{i c}-G_{o c}
$$

which involves the thermal conductance of the probe-sample system when the probe is in contact (ic index) and out of contact (oc index) with the sample, respectively. The heat input $P$ is not punctual but distributed over the volume of the electrical sensor (see Fig. 7(b)), which results in the fact that the average probe temperature is not equal to that of the apex. We introduce the thermal conductance of the probe $G_{p}$ associated with heat conduction in the cantilever. The thermal conductance at the tip-sample thermal contact $G_{c-g}$ is associated with heat exchange through the mechanical contact, the water meniscus due to capillary condensation around the mechanical contact, and direct tip-sample transfer by the gas close to the apex. $G_{s}$ is the thermal conductance associated with heat dissipation in the sample and $G_{c c-o c}$ is the thermal conductance associated with the heat losses to the environment (conduction or convection in the gas however, radiative effects can be neglected). Contrarily to [46] we consider the same thermal resistance network (Fig. 7) to describe the probe-sample system for both the Wollaston and Pd probes used. One can draw two equivalent schematics, where the first one represents the real situation and the second, fictitious one involves a factor $K$ so that $\theta_{p}^{a p e x}=K . \bar{\theta}_{p}$. This factor hardly changes between the out-of-contact and in-contact positions. Out of contact, it is found analytically to be 1.5 for the Wollaston probe. Numerical simulation by FEM for the Pd probe provide a value of 1.42 in the same situation [42]. The temperature profile changes slightly at the very apex when in contact, which can be embedded in $R_{c-g}$. The reader is referred to [47] for more details on the probe analysis. From the measurement $\Delta G_{p-s}$ is associated with the global probe-sample heat transfer and is given by:

$$
G_{i c}=\frac{P_{e l-i c}}{\theta_{p, i c}^{a p e x}}=G_{p}+\frac{G_{s} G_{c-g}}{G_{s}+G_{c-g}}
$$

and

$$
G_{o c}=\frac{P_{e l-o c}}{\theta_{p, o c}^{a p e x}}=G_{p}+G_{c c-o c}
$$

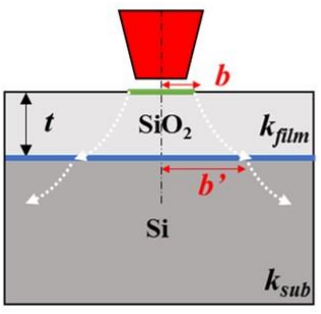

(a)

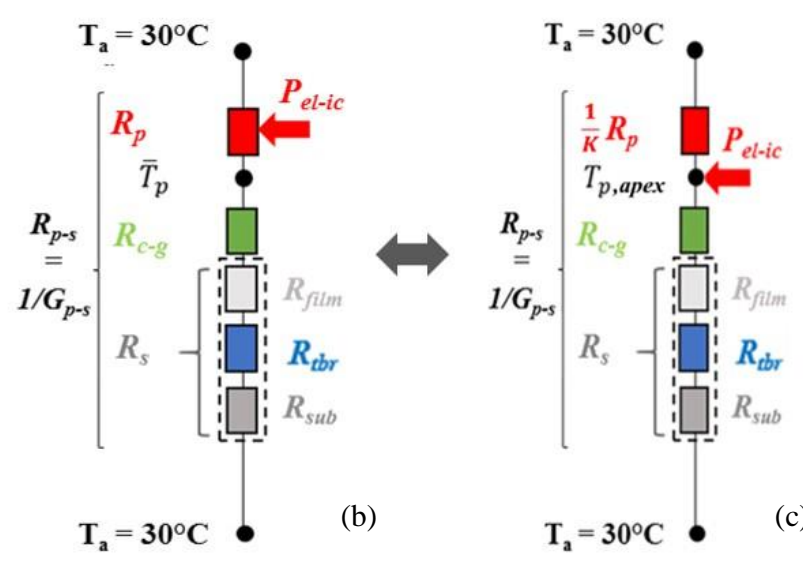

FIG. 7. Schematic of a SThM probe in contact with a thin layer on a substrate (a). The entire system consists of the thermal resistance of the probe $R_{p}$, the probe-sample thermal contact resistance $R_{c-g}$ and thermal resistance in the sample $R_{s} . R_{s}$ is the sum of the thermal resistance of the thin layer $R_{\text {film }}$, the thermal resistance of the thin layer/substrate boundary $R_{t b r}$, the ballistic resistance $R_{b a l}$ and the thermal resistance in the substrate $R_{\text {sub }}$. Real situation (b) and fictitious situation that involves a factor K so that $\theta_{p}^{a p e x}=K \cdot \bar{\theta}_{p}$ (c). 
Note that in Eq. (14) we do not consider losses to the environment. It is now well understood that the losses are not constant neither when the probe is far from the sample or close to it. On top of that, the sample attracts the heat flowing out of the tip, capturing the heat flux lines (possibly inducing a flow of heat into the sample over a large micrometric area) so that in the end $G_{c c-i c} \approx$ 0 . From Eqs. (13-15) the thermal conductance of the sample can be determined from experiment as:

$$
G_{S}=\frac{G_{c-g}\left(\Delta G_{p-s}+G_{c c-o c}\right)}{G_{c-g}-\Delta G_{p-s}-G_{c c-o c}}
$$

where $G_{c c-o c}$ is estimated from calibration of the probe under air and vacuum conditions.

If heat dissipation into a bulk sample of thermal conductivity $k_{s}$ is diffusive, $G_{s}$ is also given by Eq. (6). Note that while the temperature profile on top of the sample may be complex, as a result of the various heat transfer channels between the probe and the sample, it is customary to consider a single radius, which can be obtained by averaging as in Sec. II.B. In this case, it is straightforward to plot $\Delta G_{p-s}$ according to $k_{s}$ :

$$
\Delta G_{p-s}=\frac{G_{c-g}}{1+\frac{G_{c-g}}{4 . b . k_{s}}}-G_{c c-o c}
$$

which is a curve of shape close to $A /\left(1+\frac{B}{k_{s}}\right)+C$. The parameters $A, B$ and $C$ can be determined from a best fit of $\Delta G_{p-s}$ experimental values obtained on bulk reference samples (obtained under the same environmental conditions as the measurements being performed). It is important to note that the identification $\left(A=G_{c-g}, B=\frac{G_{c-g}}{4 . k_{s}}\right.$, $C=-G_{c c-o c}$ ), which has traditionally been made in SThM, is based on the assumptions that (i) $b$ and $G_{c-g}$ are invariant with $k_{s}$ and that (ii) ballistic effects do not operate within the reference samples or can be embedded in $G_{c-g}$ due to sample independency. These assumptions can be questioned in relation to studies [17, 19, 23-26]. that have demonstrated that, in air, $b$ and $G_{c-g}$ vary as a function of $k_{s}$. Moreover, ballistic effects should be present in crystalline samples with high thermal conductivity. In this situation $b$ reaches values of the same order of magnitude, or lower than the averaged mean free path of energy carriers in the sample material. In addition, $G_{c-g}$ is dependent on sample surface parameters such as roughness, as well as the probe and sample material contact resistance, which vary for each reference sample. Consequently, care should be taken when analyzing the physical meaning of $(A, B, C)$. In the following, we initially consider the calibration curve $\Delta G_{p-s}=f\left(k_{s, e f f}\right)$ as a way to determine $k_{s, e f f}$ without requiring the various thermal conductances to be identified. Note also that such a curve should be obtained for each probe used.

Interestingly, the film-on-substrate sample proposed allows the contribution of the probe-sample thermal contact to be considered as constant as the surface material does not change and the roughness (lower than $1 \mathrm{~nm}$ ) does not vary significantly at each site. It is also reasonable to assume that changes of the thermal contact due to possible changes in the elastic modulus of the sample with the $\mathrm{SiO}_{2}$ thickness are negligible. Eq. (16) is combined with Eq. (11), resulting in the network schematized in Fig. 7. One can associate an effective thermal conductivity to the $\{$ film+substrate system $\}$ so that $G_{s}=4 . b . k_{s, e f f}$. This is possible because the thermal contact radius $b$ is determined by the sample effective conductance: the material architecture does not play any role [14].

Once the effective thermal conductances have been determined, the sample model given in Eq. (11), where $b$ is the only unknown, can be applied. The hope is that the smoothing associated with the numerical determination of $(A, B, C)$ prior to the determination of $k_{s, \text { eff }}$ decreases the impact of the variability of $G_{c-g}$. The thermal radius is determined as follows:

$$
\begin{gathered}
b=1 /\left(4 . k_{\text {s,eff }} \cdot\left(R_{\text {film }}+R_{\text {int }}+R_{\text {sub-ballistic }}+\right.\right. \\
\left.\left.R_{\text {sub-diffusive }}\right)\right),
\end{gathered}
$$

where all the terms depend on the thickness $t$. The thermal contact conductance $G_{c-g}$, identical for all samples, can also be deduced from a measurement of $\Delta G_{p-s}$ with a similar material, e.g. bulk $\mathrm{SiO}_{2}$.

\section{EXPERIMENTS AND RESULTS}

\section{A. Determination of effective thermal conductivities}

SThM images of the mosaic of $\mathrm{SiO}_{2}$ steps were performed in ambient air conditions with the two types of probe. The probes used were heated to $\theta=70 \mathrm{~K}$ for the Wollaston probe and $\theta=65 \mathrm{~K}$ for the Pd probe while they had no interaction with the sample (out of contact conditions). Next the probes were brought into contact with the sample and scanned. Figs. 8(a) and 9(a) show thermal images of $\Delta G_{p-s}$ obtained using the Wollaston probe and the Pd probe, respectively. The images are composed of (256 x 256) pixels and each pixel corresponds to $(300 \times 300) \mathrm{nm}^{2}$. The acquisition time for each pixel was $4 \mathrm{~ms}$ for the Wollaston probe and $11 \mathrm{~ms}$ for the Pd probe, larger than their time constants. Based on these images, and to avoid topography artifacts that are observed at the edges of the steps, the average signal of $\overline{\Delta G_{p-s}}$ for each film thickness was calculated in the center of each step over an area of $(15 \mathrm{x} 15) \mu \mathrm{m}^{2}$. $\overline{\Delta G_{p-s}}$ is represented as a function of $t_{\mathrm{SiO} 2}$ in Figs. 8(b) and 9(b) for the Wollaston and the Pd probes, respectively. In these figures the dispersion of $\overline{\Delta G_{p-s}}$ corresponds to the rms values, which are relatively small and not always visible on the plots. As expected the larger the oxide thickness $t_{S i O 2}$, the lower the measured thermal conductance $\overline{\Delta G_{p-s}}$. Using the calibration of each probe, the effective thermal conductivity as a function of $t_{S i O 2}$ can be determined. Results are shown in Fig. 8(c) and Fig. 9(c). 

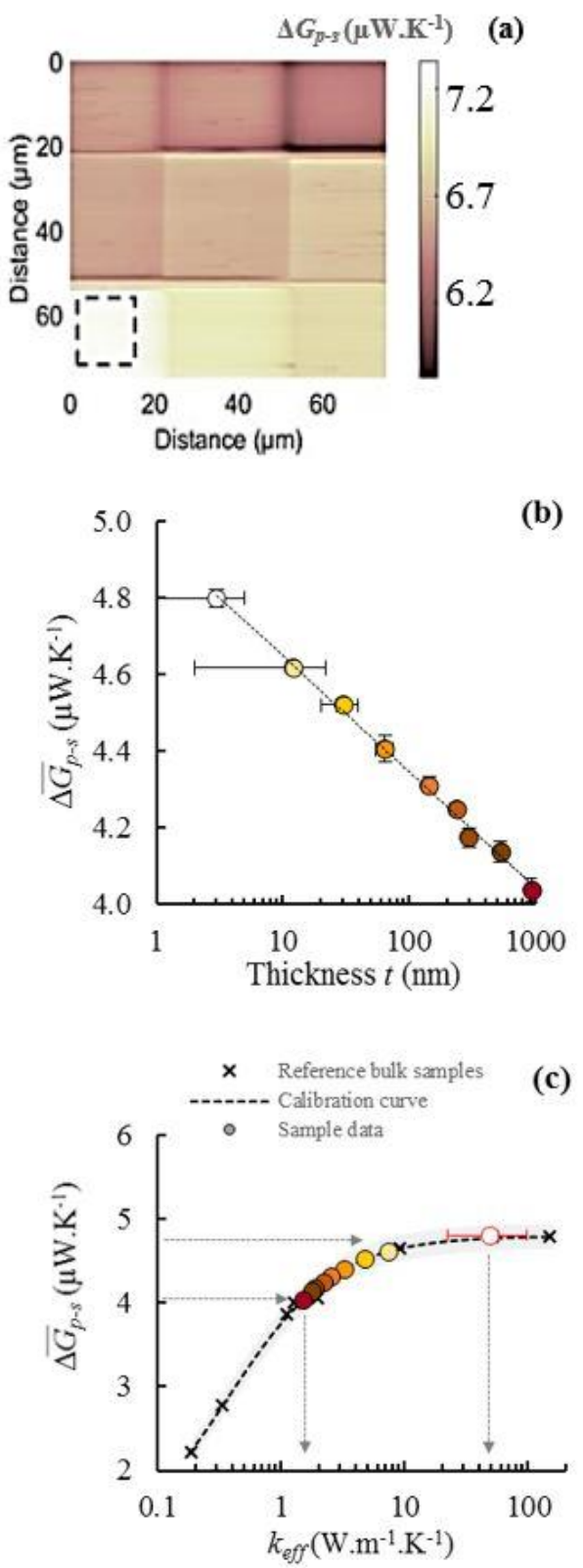

FIG. 8. (a) SThM image of the probe-sample thermal conductance $\Delta G_{p-s}$ obtained with the Wollaston probe. The orientation of the sample is the same as in Fig. 1. (b) Value of the mean signal $\overline{\Delta G_{p-s}}$ (measured on a surface (15 $\times 15) \mu \mathrm{m}^{2}$ as indicated by the black dotted square in (a) as a function of the $\mathrm{SiO}_{2}$ thickness. Figure (b) sets the correspondence between coloured circles and the SiO2 film of different thicknesses for following figures. (c) Use of the Wollaston calibration curve deduced from measurements on reference bulk samples for evaluating the effective thermal conductivity $k_{\text {eff }}$ of each step. The grey area represents the dispersion of the measurement around the mean value of $\Delta G_{p-\text { s. }}$.

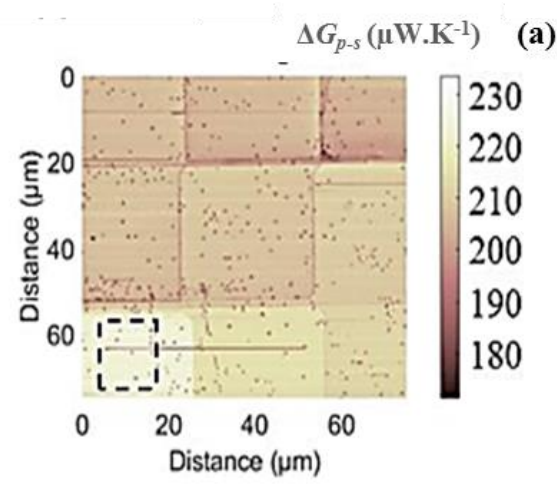

(b)
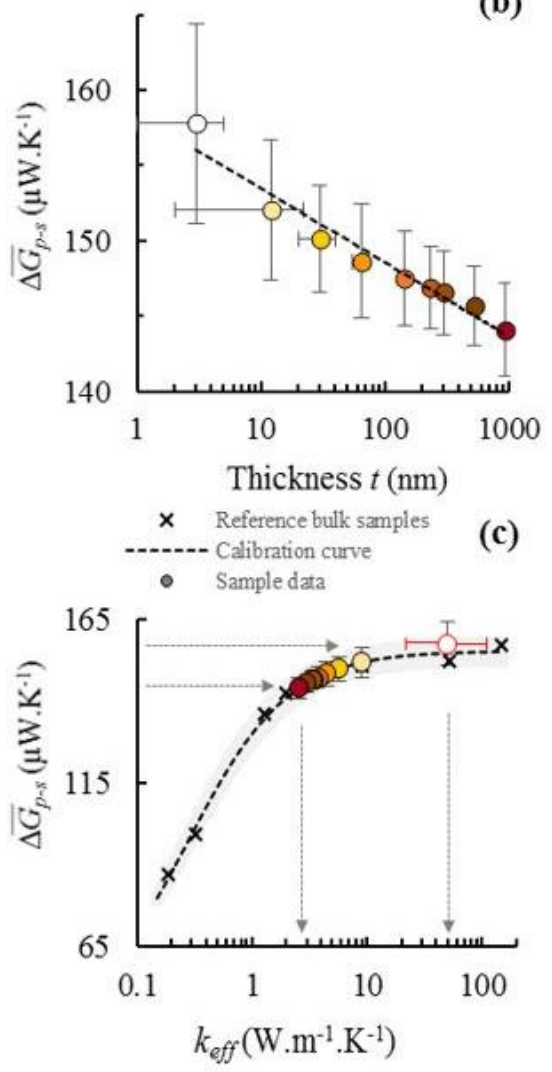

FIG. 9. (a) SThM image of the probe-sample thermal conductance $\Delta G_{p-s}$ for obtained with the Pd probe. The orientation of the sample is the same as in Fig. 1. (b) Value of the mean signal $\overline{\Delta G_{p-s}}$ (measured on a surface $(15 \times 15)$ $\mu \mathrm{m}^{2}$ as indicated by the black dotted square in (a) as a function of the $\mathrm{SiO} 2$ thickness. (c) Use of the Pd probe calibration curve deduced from measurements on reference bulk samples for evaluating the effective thermal conductivity $k_{\text {eff }}$ of each step. The grey area represents the dispersion of the measurement around the mean value of $\Delta G_{p-s}$. 
For the Wollaston probe (Fig 8(b)) it appears that $\overline{\Delta G_{p-s}}$ varies logarithmically as a function of the film thickness. For the thickest step, $\overline{\Delta G_{p-s}}$ is almost the same as that measured on the $\mathrm{SiO}_{2}$ calibration sample, with values of $1.28 \mathrm{~W} \cdot \mathrm{m}^{-1} \cdot \mathrm{K}^{-1}$ on bulk $\mathrm{SiO}_{2}$ and $1.1 \mathrm{~W} \cdot \mathrm{m}^{-1} \cdot \mathrm{K}^{-1}$ on the oxide step sample (Fig. 8(c)). The measurement point for the lowest $\mathrm{SiO}_{2}$ thickness (point surrounded in red) is not in the sensitive range of the calibration curve (low effective thermal conductivities), so the exact effective thermal conductivity $k_{\text {eff }}$ for this point cannot be estimated. It can only be said that for $t_{\mathrm{SiO} 2}=7$ $\mathrm{nm} k_{\text {eff }}$ is greater than tens of W. $\mathrm{m}^{-1} \cdot \mathrm{K}^{-1}$. Thermal conductivity values can be determined from a calibration range of about $8 \mathrm{~W} \cdot \mathrm{m}^{-1} \cdot \mathrm{K}^{-1}$ for $t_{\mathrm{SiO} 2}=12 \mathrm{~nm}$ to about $1.5 \mathrm{~W} \cdot \mathrm{m}^{-1} \cdot \mathrm{K}^{-1}$ for $t_{\mathrm{SiO} 2}=950 \mathrm{~nm}$.

For the Pd probe, the decrease of $\overline{\Delta G_{p-s}}$ as a function of $t_{\mathrm{SiO} 2}$ is also logarithmic (Fig. 9(b)), although the experimental points are more dispersed around the fitting curve. The correspondence with the calibration curve, shown in Fig. 9(c), shows that the measurement point for the lowest thickness (point surrounded in red) is also in the area of insensitivity to thermal conductivity for this probe. So for $t_{\mathrm{SiO} 2}=7 \mathrm{~nm}$ a loose lower bound of $\sim 30 \mathrm{~W} \cdot \mathrm{m}^{-1} \cdot \mathrm{K}^{-1}$ can be underlined. Finally, the effective thermal conductivity $k_{e f f}$ measured with the Pd probe ranges from $2 \mathrm{~W} \cdot \mathrm{m}^{-1} \cdot \mathrm{K}^{-1}$ for the largest thickness to more than $30 \mathrm{~W} \cdot \mathrm{m}^{-1} \cdot \mathrm{K}^{-1}$ for the lowest one.

Finally, identified effective conductivity values for both probes are summarized in Fig. 10, where they are plotted according to the film thickness. Equating of $\overline{\Delta G_{p-s}}=f(A, B, C)$ and the logarithmic evolution of $\overline{\Delta G_{p-s}}$ as a function of the thickness suggests a functional dependency of $k_{\text {eff. }}$ One finds that $k_{\text {eff }}$ vs. $t$ can be relatively-well fitted with the following expression:

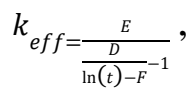

where $D, E$ and $F$ are constants depending on the probe used.

\section{B. Thermal contact radii and probe parameters}

Fig. 11 represents the thermal radius $b$ as a function of effective thermal conductivity $k_{\text {eff. }}$ It is obtained from Eq. (18). The same information can be obtained in principle directly from the calibration curves. However, these results may be less sensitive to the surface conditions. Data confirm that the lower the thermal conductivity, the greater the thermal radius. That is, for a low thermal conductivity material the flow lines extend over the surface (large $b$ ), while for a conductive material in which heat flows more readily, the flow lines are more perpendicular to the surface and sink into the material (low $b)$. For the Wollaston probe, the thermal radius in ambient air decreases from $1000 \mathrm{~nm}$ for $k_{\text {eff }}=1.5 \mathrm{~W} \cdot \mathrm{m}^{-1} \cdot \mathrm{K}^{-1}$ to $200 \mathrm{~nm}$ for $k_{\text {eff }}$ larger than $3 \mathrm{~W} \cdot \mathrm{m}^{-1} \cdot \mathrm{K}^{-1}$. For the Pd probe, $b$ decreases from $2000 \mathrm{~nm}$ for $k_{\text {eff }}=2 \mathrm{~W} \cdot \mathrm{m}^{-1} \cdot \mathrm{K}^{-1}$ to $250 \mathrm{~nm}$ for $k_{\text {eff }}$ larger than $3 \mathrm{~W} \cdot \mathrm{m}^{-1} \cdot \mathrm{K}^{-1} \cdot b$ values for the nanoprobe used are surprisingly found to be larger than those obtained for the Wollaston probe used in air.

From values of thermal resistance measured experimentally in ambient air $\overline{\Delta G_{p-s}}$ (see Eq. (17)) and by using the model (Eq. (11)), it is possible to estimate the thermal contact resistance $R_{c-g}$ between the probe and the sample. For the Wollaston probe, $R_{c-g}$ is larger than $10^{5}$ W.K. ${ }^{-1}$. For the Pd probe, the estimated $R_{c-g}$ value is of (46.6 \pm 1.6). $10^{5} \mathrm{~K} . \mathrm{W}^{-1}$.

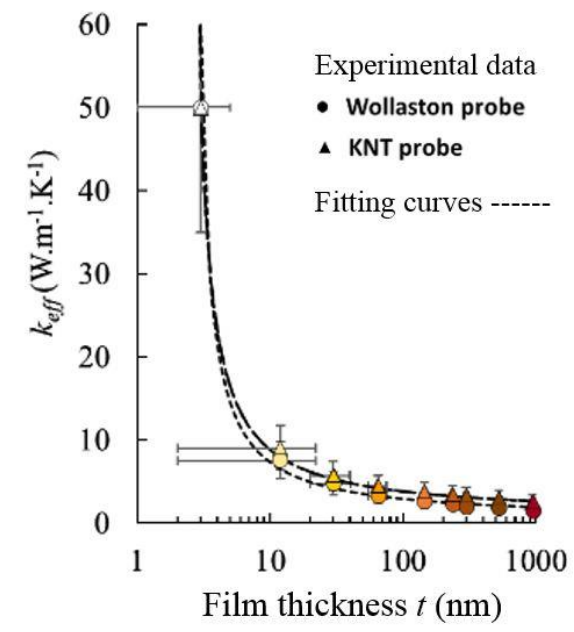

FIG. 10. Effective thermal conductivity $k_{\text {eff }}$ estimated using calibrated Wollaston and $\mathrm{Pd}$ probes according to the thickness $t$ of the $\mathrm{SiO}_{2}$ sample steps.
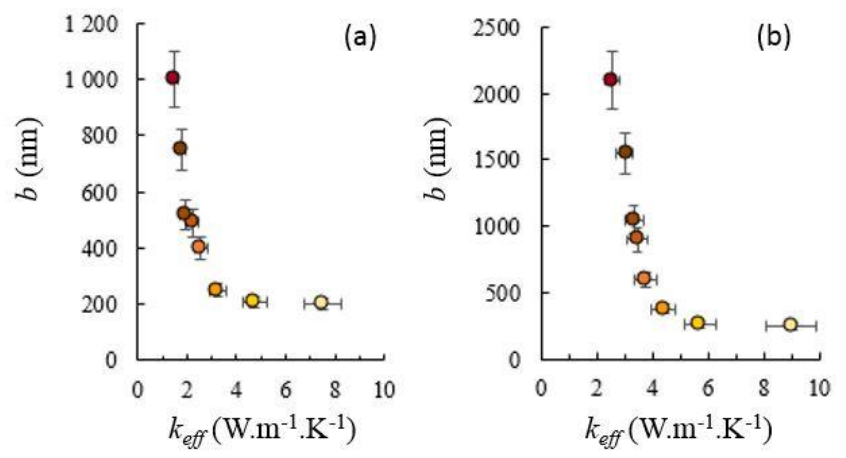

FIG. 11. Thermal radius $b$ as a function of the sample step effective thermal conductivity $k_{\text {eff }}$ for the Wollaston (a) and the $\mathrm{Pd}(\mathrm{b})$ probes used.

\section{DISCUSSION}

\section{A. Sensitivity to inhomogeneity in depth}

Our results show that in ambient air, the Wollaston and Pd probes are sensitive to the decrease in thermal conductance measured over the entire range tested $t \in[3-1000] \mathrm{nm}$. That is, the thermal signal is sensitive in depth up to a distance of at least $1000 \mathrm{~nm}$ for both probes used in this range of effective thermal conductivities.

In spherical coordinates, heat diffusion predicts a $1 / r$ decay of the temperature field away of the heat source. As a result, the sample thermal conductance is approximately proportional to $b^{2} \times(1 / b-1 / D)$ if $D$ is an isothermal cold bath of hemispherical shape centered around the source of sufficient size $2 b$. This suggests that 
an object of size $L$ located at $d$ from the heat source impacts the power dissipated with respect to a bulk substrate at best with a change of b.d.f(L/d), where the first factor accounts for the deviation due to an hemispherical perturbation and the second one for the fact that the finite size of the object impacts only a limited solid angle. For a flat substrate below the film $d \sim t$ and $f\left(\frac{L}{d}\right)=$ $O$ (1), so the substrate below films of thickness $t$ can be resolved if relative variation of thermal conductance $b / t$ can be measured. This suggests sensitivity to depth much larger than $1 \mu \mathrm{m}$ when there is a strong contrast of thermal conductivity as numerically shown in [48]. The trends of Figs. 8(b), 9(b) (no sign of levelling off in logarithmic scale) and the radii larger than hundreds of nanometers shown in Fig. 11, appear compatible with a sensitivity to depth larger by one order of magnitude [12].

In contrast, measurements made with a Pd probe under primary vacuum show that from a thickness of about $250 \mathrm{~nm}$ (Fig. 12) the probe-sample thermal conductance, which is one order smaller than under ambient air conditions, becomes constant and it is no longer possible to differentiate the different thicknesses. The volume probed is therefore smaller under vacuum than that probed under ambient air due to a thermal contact surface, which is smaller in vacuum than in air.

Note that another popular SThM method is based on the difference of thermal conductance $\Delta G_{p-s}^{j u m p}$ before and after the probe jump into contact with the sample under ambient air [49, 50]. This allows the effect of the contact (mechanical+meniscus) to be singled out without requiring vacuum conditions, and is therefore subject to the same limitations as that in vacuum. It is therefore expected that the sensitivity to depth would also be limited to few hundreds of nanometers for the Pd probe, similar that shown in Fig. 12.

In addition, this work also confirms that native oxide can affect SThM measurements [14]. Indeed both the Wollaston and Pd probes in our experiments detect the variation in effective thermal conductance (Figs. 8(b) and 9(b)) generated by the thinnest oxide thickness of a few nanometers for the $\mathrm{SiO}_{2}$ steps sample, which is comparable to a native oxide film of around 1-2 $\mathrm{nm}$ that naturally appears under ambient air on the surfaces of certain metals or semiconductors.

\section{B. Sensitivity to effective thermal conductivity}

The calibration curves (Figs. 8(c) and 9(c)) level off at large thermal conductivities. As a result, the uncertainty of the determined effective thermal conductivities becomes very large for values above $5 \mathrm{~W} \cdot \mathrm{m}^{-1} \cdot \mathrm{K}^{-1}$, and are already significant above $3 \mathrm{~W} \cdot \mathrm{m}^{-1} \cdot \mathrm{K}^{-1}$. This uncertainty is mostly due to dispersion around the fitting curve observed for the bulk samples, which comes from the surface effects and partly-ballistic transport which impact the materials. The current work indicates that SThM is sensitive to much higher thermal conductivities (high repeatability and relatively small dispersion of $\Delta G_{p-s}$ ), maybe almost up to the value of silicon, but the issue is that the calibration curve is not accurate enough to exploit this sensitivity. This calls for improved calibration curves.

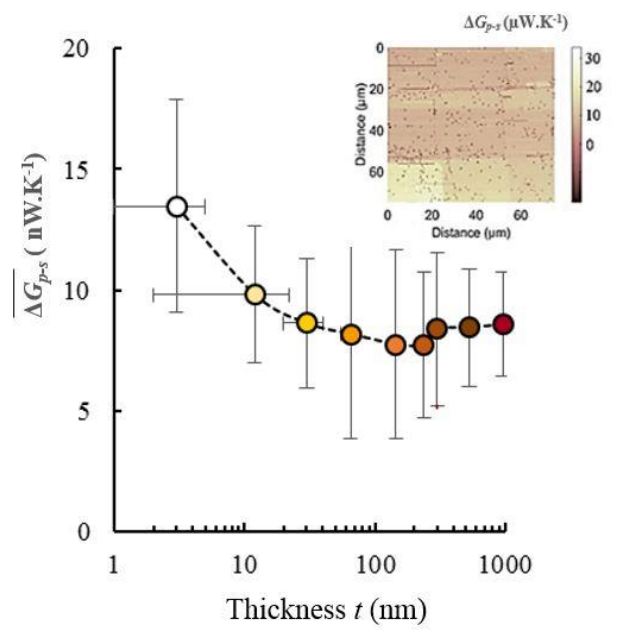

FIG. 12. Probe-sample thermal conductance $\overline{\Delta G_{p-s}}$ as a function of the $\mathrm{SiO}_{2}$ thickness for a Pd probe operated in vacuum. The data is averaged over a surface of $(15 \times 15) \mu \mathrm{m}^{2}$. Error bars represent the dispersion of the measurement around the mean value of $\overline{\Delta G_{p-s}}$.

The present mosaic samples could play the role of the samples with different effective thermal conductivities, provided that the values are not determined by application of the bulk-reference calibration curves but with another manner. In addition, the ideal sample for calibration of SThM probes could be based on films with thermal conductivity ten to one hundred times lower than $\mathrm{SiO}_{2}$ as it is known that SThM probes are particularly sensitive to thermal conductivity lower than $1.5 \mathrm{~W} \cdot \mathrm{m}^{-1} \cdot \mathrm{K}^{-1}[6]$.

\section{CONCLUSIONS}

The proposed oxide steps sample provides a series of nine surfaces made of the same material, with identical roughness, but with different effective thermal conductivities ranging over more than one order of magnitude. This allows the SThM sensitivity to be measured within a given set of operating conditions, removing the influence of factors such as tip-sample contact resistance and tipsample contact area that can mask the true sensitivity. A model has been provided to determine the thermal contact area and contact thermal resistance from the experimental data. Note that the mentioned factors change dynamically with wear of the probe apex and one needs to quantify the degradation of the probe before and after scanning. By repeating measurements on the different mosaic elements regularly, the proposed sample provides a simple way to analyze the evolution of the SThM calibration curve with use.

Key benefits are the possibilities that the proposed sample offers measurement of the sensitivity of a SThM system, as well as a comparison of the depth and spatial resolution of different probes. Used with the proposed modeling it allows comparison of the performance of different types of SThM probes.

The numerical application of the proposed modelling shows that ballistic conduction should be taken into account in crystalline 
substrates below ultrathin films, also for those made of amorphous materials. For $\mathrm{SiO}_{2}$ layers of thickness lower than $200 \mathrm{~nm}$ on a $\mathrm{Si}$ substrate, results show that there is ballistic conduction in the substrate when the thermally heated surface has a radius smaller than the thickness. While this is moderately important under ambient air conditions, it is of key importance for vacuum.

\section{ACKNOWLEDGMENTS}

This work has received funding from the European Union Seventh Framework Program FP7-NMP-2013-LARGE-7 under GA No. 604668 Project QUANTIHEAT. We thank D. Renahy for early work and A. Pic for discussion.

\section{DATA AVAILABILITY STATEMENT}

The data that support the findings of this study are available from the corresponding author upon reasonable request.

\section{REFERENCES}

[1] Ch. Dames, Annual Review of Heat Transfer 16 (2013).

[2] E. Pop, D. A. Mann, K. E. Goodson, and H. Dai, Journal of Applied Physics, 101(9), 093710 (2007).

[3] T. Phan, S. Dilhaire, V. Quintard, W. Claeys, and J. Batsale, Microelectron. J. 29, 181-90 (1998).

[4] D. I. Florescu, V. M. Asnin, F. H. Pollak, R. J. Molnar, and C. E. C. Wood, J. Appl. Phys. 88(6), 3295-3300 (2000).

[5] Y. Hu, L. Zeng, A. Minnich, and S. D. Mildred. Nature Nanotech 10, 701-706 (2015).

[6] S. Gomès, A. Assy, and P. O. Chapuis, physica status solidi (a), 212(3), 477-494 (2015).

[7] W. Jeong, S. Hur, E. Meyhofer and P. Reddy, Nanoscale and Microscale Thermophysical Engineering, 19(4), 279-302 (2015).

[8] Y. Zhang, W. Zhu, F. Hui, M. Lanza, Th. Borca-Tasciuc M. Muñoz Rojo, Advanced functional materials, 30(18), 1900892 (2020).

[9] F. Menges, H. Riel, A. Stemmer, C. Dimitrakopoulos, and B. Gotsmann, Phys. Rev. Letters, 111(20), 205901 (2013).

[10] S. Pailhes, H. Euchner, V. M. Giordano, R. Debord, A. Ass, S. Gomès, A. Bosak, D. Machon, S. Paschen and M. De Boissieu, Phys. Rev. Letters, 113(2), 025506 (2014).

[11]P. Newby, B. Canut, J. M. Bluet, S. Gomès, M. Isaiev, R. Burbelo, K. Termentzidis, P. Chantrenne, L. Frechette, and V. Lysenko, J. Appl. Phys. 114(1), 014903-9 (2013).

[12] S. Gomès, L. David, V. Lysenko, A. Descamps, T. Nychyporuk, and M. Raynaud, J. Phys. D: Appl. Phys. 40, 6677-6683 (2007)

[13] J. L.Bosse, M. Timofeeva, P. D. Tovee, B. J. Robinson, B. D. Huey, and O. V. Kolosov, Journal of Applied Physics, 116(13), 134904 (2014).

[14] 8 A. M. Massoud, J. M. Bluet, V. Lacatena, M. Haras, J. F. Robillard, and P. O. Chapuis, Appl. Phys. Letters 111(6), 063106 (2017).

[15]E. Puyoo, S. Grauby, J. M. Rampnoux, E. Rouvière, and S. Dilhaire, J. Appl. Phys. 109(2), 024302 (2011).

[16] JCGM 2012 International Vocabulary of Metrology-Basic and General Concepts and Associated Terms (VIM) 3rd edn) (www.bipm.org/utils/common/documents/jcgm/JCGM_200_2012.pdf)

[17] A.A. Wilson and T. Borca-Tasciuc, Rev. Sci. Instrum. 88, 074903 (2017).

[18]B. D. Iverson, J. E. Blendell, and S. V. Garimella, Note (2010). RSI 81, 036111 (2010).

[19] S. Lefevre, S. Volz, J. B. Saulnier, C. Fuentes, and N. Trannoy, RSI 74(4), 2418-2423 (2003).
[20]E. Guen, P.-O. Chapuis, N. J. Kaur, P. Klapetek, and S. Gomès, Impact of roughness on heat conduction involving nanocontacts, submitted to APL 2020.

[21] S. Lefèvre, and S. Volz, RSI 76(3), 033701 (2005).

[22]E. Puyoo, Ph. D Thesis (Doctoral dissertation of Bordeaux, 2010). http://www.theses.fr/2010BOR14110

[23] L. David, S. Gomès, and M. Raynaud, J. Phys. D: Appl. Phys. 40, 43374346 (2007).

[24]A. Assy, Ph. D Thesis (Doctoral dissertation of Lyon, 2015). http://www.theses.fr/2015ISAL0001

[25] M. Massoud, Ph. D Thesis (Doctoral dissertation of Lyon, 2016). http://www.theses.fr/2016LYSEI063

[26] T. P. Nguyen, L. Thiery, S. Euphrasie, S. Gomès, B. Hay, and P. Vairac, RSI 90(11), 114901 (2019).

[27] R. B. Dinwiddie, RR. J. Pylkki, and P. E. West, Thermal conductivity 22, 668-668 (1993).

[28]P. S. Dobson, J. M. Weaver, and G. Mills, Sensors IEEE, 708-711 (2007).

[29] M. Hinz, O. Marti, B. Gotsmann, M. A. Lantz, M. A., and U. Dürig, Appl. Phys. Letters 92(4), 043122 (2008).

[30] A. Al Mohtar, G. Tessier, R. Ritasalo, M. Matvejeff, J. StormonthDarling, P. S. Dobson,... and J. P. Roger, Thin Solid Films 642, 157-162 (2017).

[31] M. M. Yovanovich, J. R. Culham, and P. Teertstra, IEEE Trans. Components Packag. Manuf. Technol. Part A 21(1), 168-176 (1998).

[32] J. R. Dryden, J. Heat Transfer 105(2), 408 (1983).

[33] Y.S. Muzychka, M.R. Sridhar, M.M. Yovanovich, and V.W. Antonetti, J. Thermophys. Heat Transf. 13(1) (1999).

[34]F. Menges, H. Riel, A. Stemmer, C. Dimitrakopoulos, and B. Gotsmann, Phys. Rev. Lett. 111, 1 (2013).

[35] The average mean free path $\Lambda$ sub can be determined by inverting the spectral kinetic expression of thermal conductivity $\frac{k}{\frac{1}{3}\left\langle c_{p} v\right\rangle}$, with $\langle c v\rangle$ $=\sum_{p} \int_{0}^{\omega_{\max }} c_{p}(\omega) v_{g, p}(\omega) d \omega$ where $c\left(c_{p}\right)$ is the (polarizationdependent spectral) heat capacity and $v_{(g, p)}$ the (polarization-dependent spectral) velocity of the phonons in the isotropic dispersion assumption. Depending on the fit of the dispersion curve, the value is close to 170 $185 \mathrm{~nm}$. Here, we take $\Lambda$ sub $=170 \mathrm{~nm}$.

[36] G. Wexler, Proc. Phys. Soc. 89(4), 927-941 (1966).

[37] Y. Sharvin, Sov. Phys. JETP, 21, 655 (1965).

[38]B. Nikolić and P. B. Allen, Physical Review B, 60(6), 3963 (1999).

[39] A. Assy, S. Lefèvre, P. O. Chapuis, and S. Gomès, J. Phys. D: Appl. Phys. 47(44), 442001 (2014).

[40] S. Lefèvre, J. B. Saulnier, C. Fuentes, and S. Volz, Superlattices Microstruct., 35(3-6), 283-288, (2004).

[41] S. Lefèvre, Ph. D Thesis (Doctoral dissertation of Lyon, 2004).

[42]E. Guen, Ph. D Thesis (Doctoral dissertation of Lyon, 2020).

[43]P. Tovee, M. Pumarol, D. Zeze, K. Kjoller, and O. Kolosov, J.of Appl. Phys. 112(11), 114317 (2012).

[44] A. Assy, and S. Gomès, Nanotechnology 26(35), 355401 (2015).

[45]C. T. Gibson, G. S. Watson, and S. Myhra, Nanotechnology 7(3), 259 (1996).

[46] M. Chirtoc, J. Bodzenta and A. Kaźmierczak-Bałata, International Journal of Heat and Mass Transfer, 156, 119860 (2020).

[47]A. Alkurdi, E. Guen, et al., Resistive-thermometry scanning thermal microscopy: combined experimental/numerical approaches for thermal studies of nano-objects, J. Phys. Cond. Mat., to appear

[48] J. Bodzenta, J. Juszczyk, A. Kaźmierczak-Bałata, P. Firek, A. Fleming, and M. Chirtoc, International Journal of Thermophysics, 37(7), 73 (2016).

[49] K. Kim, J. Chung, G. Hwang, O. Kwon and J. S. Lee, Acs Nano, 5(11), 8700-8709 (2011).

[50] J. Spiece, C. Evangeli, K. Lulla, A. Robson, B. Robinson, B., O. Kolosov, Journal of Applied Physics, 124(1), 015101 (2018). 